\title{
High-resolution imaging by multiple-image deconvolution
}

\author{
M. Bertero, P.Boccacci, G. Desiderà, and G. Vicidomini \\ DISI, Università di Genova, Via Dodecaneso 35, I-16146, Genova, Italy
}

\begin{abstract}
Image deconvolution is a powerful tool for improving the quality of images corrupted by blurring and noise. However, in some cases, the imaging system is affected by anisotropic resolution, i. e. the resolution depends on the direction in the imaging plane or volume. Such a distortion cannot be corrected by image deconvolution. One example, from Astronomy, is the Large Binocular Telescope (LBT) under construction on the top of Mount Graham (Arizona). A second example, from Microscopy, is the confocal microscope. In both cases, the situation can be improved if different images of the same target can be detected by rotating the instrument or by rotating the target. Then the problem arises of obtaining a unique high-resolution image from different images taken at different orientation angles. Such a problem is called multiple-image deconvolution.

In this paper, after a brief illustration of the two examples mentioned above, the problem of multiple-image deconvolution is formulated and preliminarily investigated in a continuous setting (all directions are available), showing that, while resolution is anisotropic in the multiple images, it becomes isotropic in the reconstructed image. Next, methods and algorithms for the solution of the problem are presented and their accuracy illustrated by means of the results of a few numerical experiments. Finally, the possibility of a further improvement of resolution by means of superresolving methods is briefly discussed and demonstrated.
\end{abstract}

Keywords: Image reconstruction, iterative methods, super-resolution

PACS: $42.30 . \mathrm{WB}, 02.30 . \mathrm{Zz}$

\section{INTRODUCTION}

In many applications of imaging science images are corrupted by blurring and noise. If the point spread function (PSF) of the instrument is known, and if we assume for simplicity that the PSF is space invariant, then the image is given by the convolution of the object with the PSF and one can attempt to correct for the disturbing effect of the PSF by solving the so-called deconvolution problem. The latter is ill-posed, i. e. the solution is strongly corrupted by the noise perturbing the data. Then, a classical approach consists in a variational reformulation of the problem, derived from the knowledge of the statistical properties of the noise and, possibly, of additional information on the solution. For instance, maximum likelihood and Bayesian methods lead to the minimization of suitable functionals, and this task can be achieved, in general, by means of iterative methods. These approaches can provide satisfactory results in many circumstances. However, in some cases, the distortion introduced by the PSF can not be corrected because of its strong anisotropy. We discuss two examples of this situation, one from Astronomy and one from fluorescence Microscopy, and we indicate a way for overcoming this problem.

\footnotetext{
CP860, Information Optics: $5^{\text {th }}$ International Workshop, edited by G. Cristóbal, B. Javidi, and S. Vallmitjana

(C) 2006 American Institute of Physics 978-0-7354-0356-7/06/\$23.00
} 


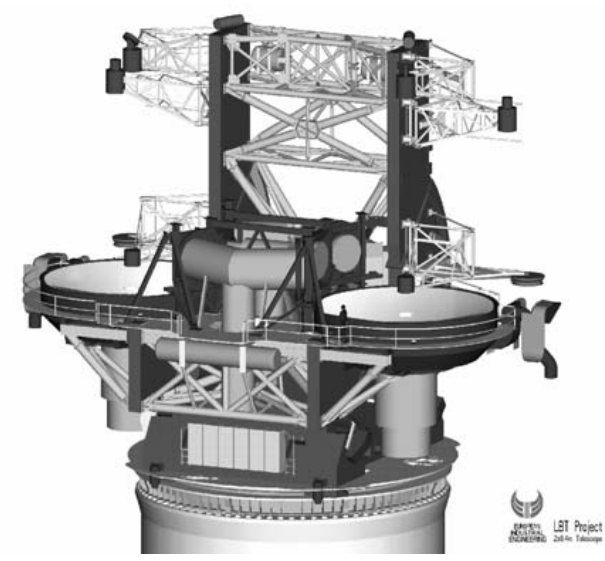

FIGURE 1. A picture of the Large Binocular Telescope

\section{EXAMPLES OF ANISOTROPIC RESOLUTION}

The first example of an optical instrument characterized by anisotropic resolution is the Large Binocular Telescope (LBT), under construction on the top of Mount Graham (Arizona) (http://medusa.as.arizona.edu/lbto/). It will consist of two $8.4 \mathrm{~m}$ mirrors on a common mount, with their centers at the distance of $14.4 \mathrm{~m}$. Atmospheric turbulence correction will be obtained with a very advanced adaptive optics (AO) system, based on pyramid sensors and adaptive secondary mirrors. The two primary mirrors have been already installed and aluminized. First light has been achieved on October 12, 2005. A picture of the telescope is shown in Fig. 1.

But the very innovative feature of LBT is that it will be equipped with a Fizeau interferometer, called LINC-NIRVANA, which will be realized by a consortium of German and Italian institutions. When it will be operating, probably mid 2008, it will provide true imaging, with the resolution of a $22.8 \mathrm{~m}$ mirror in the direction of the baseline, and the resolution of a $8.4 \mathrm{~m}$ mirror in the orthogonal direction [4]. Indeed, in the ideal case of no aberration and no atmosphere, its PSF is given by the Airy function of the $8.4 \mathrm{~m}$ mirror, modulated by the interference occurring in the direction of the baseline. In Fig. 2 we show the point spread function (PSF) of the telescope and the corresponding modulation transfer function (MTF).

The second example of an optical instrument characterized by anisotropic resolution is the confocal microscope (CM). As it is well known, 3D images can be obtained by means of this instrument, thanks to the technique known as optical sectioning: by moving the focal plane of the instrument step by step through the depth of the specimen, a series of optical sections can be recorded. In such a way, a volume image of a biological specimen can be obtained as a cube of 2D images. However the resolution along the optical axis of the microscope is much less than the resolution along the lateral directions, approximately by a factor of 3 [21]. If we consider, for instance, the epi-fluorescence mode, the section of the PSF in a plane through the optical axis (let 

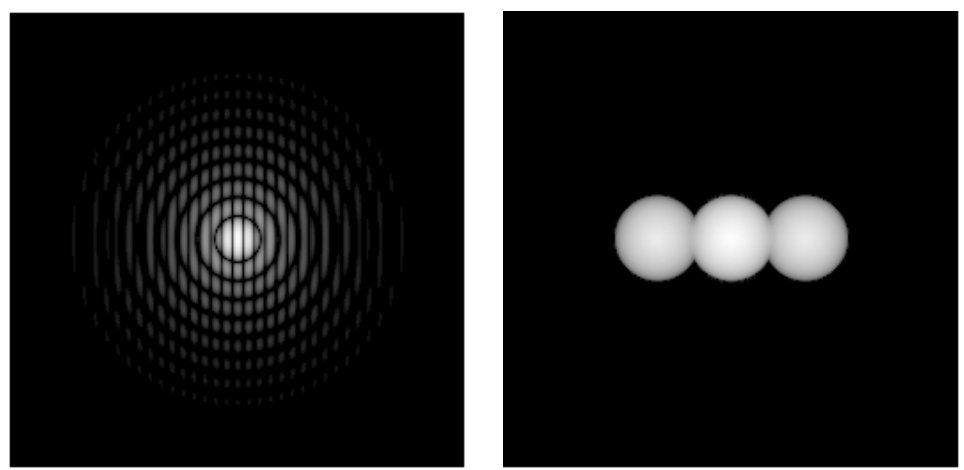

FIGURE 2. The PSF of LBT (left panel) and the corresponding MTF (right panel). In the left panel the baseline is parallel to the horizontal directiobn.
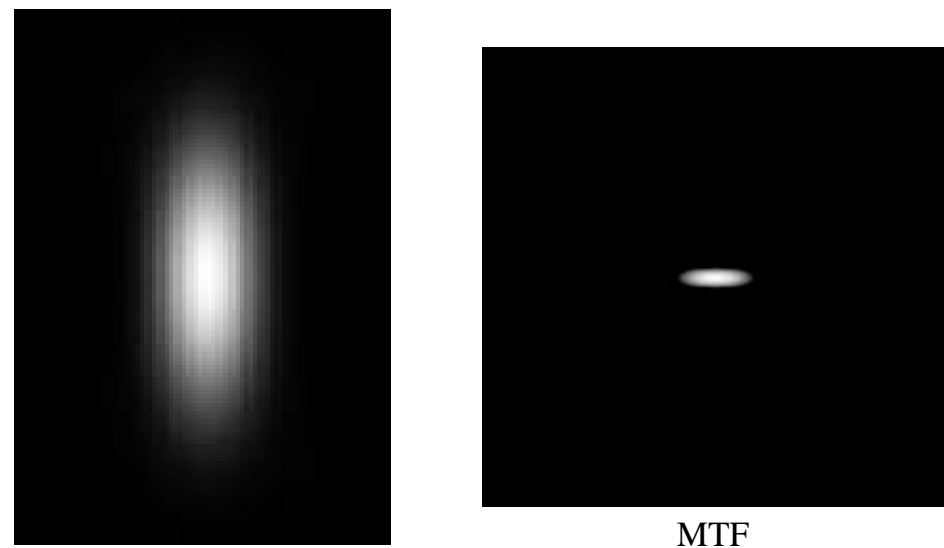

MTF

PSF

FIGURE 3. The PSF of a confocal microscope in the epi-fluorescence mode (left panel) and the corresponding MTF (right panel). In the left panel the optical axis of the microscope is parallel to the vertical direction.

us say the $x, z$-plane, where $z$ is the axial coordinate and $x$ a lateral coordinate), and the corresponding MTF are shown in Fig. 3.

In both cases, it is obvious that the anisotropy cannot be corrected by image deconvolution because it is intrinsic to the PSF of the instrument. However, in both cases, it is possible to acquire different images of the same object corresponding to different orientations of the instrument, the object being fixed, or, equivalently, to different orientations of the object, the instrument being fixed. For instance, in the case of LBT, as a consequence of Earth rotation, the instrument must rotate for keeping the astronomical object within the field of view and, as a consequence, it can acquire different images of the same object corresponding to different projections of the baseline onto the sky. On the other hand, in confocal microscopy, a technique known as micro-axial tomog- 
raphy has been introduced that consists basically in rotating the object in such a way that the axis of reduced resolution, namely the optical axis, is varied with respect to the sample $[17,10]$. In both cases, the problem arises of getting a unique reconstructed image, with possibly isotropic resolution, from the different detected images with different anisotropic resolutions. Obviously the isotropic resolution should be equal to the best resolution achieved in anisotropic images. By looking at the MTFs shown in Fig. 2 and Fig. 3, it should be obvious that this result can be achieved if the different images can assure a complete coverage in the Fourier plane (volume).

\section{MULTIPLE IMAGE DECONVOLUTION: A CONTINUOUS MODEL}

Let us denote by $\theta$ the angle between the instrument axis and a given direction in the object domain/volume. Then $K_{\theta}(\mathbf{x})$ is the corresponding PSF of the optical instrument, and $g_{\theta}(\mathbf{x})$ the corresponding detected image. $\mathbf{x}$ is a $2 \mathrm{D}$ variable in the case of Astronomy and a 3D variable in the case of Microscopy. Further, if we denote by $f$ the unknown object and we neglect noise, we have the following relationship

$$
g_{\theta}=K_{\theta} * f=A_{\theta} f, \quad \theta \in[0,2 \pi] .
$$

In this Section we consider the ideal case where the detected images are known for all values of $\theta$. Then we denote by $g$ the set of all these images (it is a one-parameter family) and by $A f$ the set of all the computed images associated to $f$

$$
g=\left\{g_{\theta}\right\}_{\theta \in[0,2 \pi]}, A f=\left\{A f_{\theta}\right\}_{\theta \in[0,2 \pi]} .
$$

Moreover, we introduce the following norm for a given set of images

$$
\|g\|^{2}=\frac{1}{2 \pi} \int_{0}^{2 \pi}\left(\int\left|g_{\theta}(\mathbf{x})\right|^{2} d \mathbf{x}\right) d \theta,
$$

while we use the standard $L^{2}$-norm for the unknown object $f$. We point out that the squared norm defined in Eq. (3) is just the mean value, over $[0,2 \pi]$, of the squared $L^{2}$ norms of the single images.

It is obvious that if we formulate the image reconstruction problem as the problem of extracting the unknown object $f$ from the set of detected images $g$, then we have redundant data, As a consequence a least squares approach looks quite natural, i. e. the reconstructed object $f_{r e c}$, can be defined as the solution of the variational problem

$$
f_{\text {rec }}=\arg \min _{f}\|A f-g\| \text {. }
$$

As it is well known, the solution of this problem is equivalent to the solution of the Euler equation

$$
A^{T} A f_{\text {rec }}=A^{T} g,
$$

and it is easy to show that the operator $A^{T} A$ is given by

$$
A^{T} A=\frac{1}{2 \pi} \int_{0}^{2 \pi} A_{\theta}^{T} A_{\theta} d \theta
$$



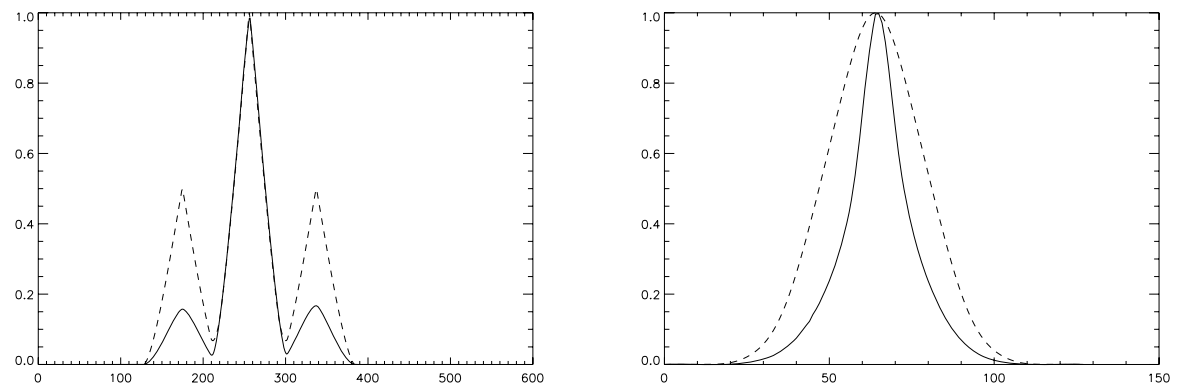

FIGURE 4. Left panel: comparison of the cut of the MTF of LBT (ideal case) along the direction of the baseline (dashed line) with the corresponding cut of the average MTF (full line). Right panel: the dashed line is the cut of the MTF of a confocal microscope along a lateral direction while the full line is again a cut of the average MTF.

where $A_{\theta}^{T}$ is the transposed (adjoint) of the operator $A_{\theta}$ with respect to the usual $L^{2}$-norm of a single image.

Since all operators $A_{\theta}$ are convolution operators, it turns out that $A^{T} A$ is also a convolution operator, with a PSF given by

$$
H(\mathbf{x})=\frac{1}{2 \pi} \int_{0}^{2 \pi}\left(K_{\theta} \otimes K_{\theta}\right)(\mathbf{x}) d \theta,
$$

where $\otimes$ denotes autocorrelation, and the corresponding MTF

$$
\hat{H}(\omega)=\frac{1}{2 \pi} \int_{0}^{2 \pi}\left|\hat{K}_{\theta}(\omega)\right|^{2} d \theta .
$$

If the PSF of the instrument does not change during the rotation (this condition is satisfied in Microscopy, but not in Astronomy where the PSF depends also on the state of the atmosphere through the AO correction, and therefore is different for different observation angles), then, by writing the MTF in polar coordinates, we find that the MTF of Eq. (8) has a circular symmetry and is given by

$$
\hat{H}(|\omega|)=\frac{1}{2 \pi} \int_{0}^{2 \pi}|\hat{K}(|\omega|, \theta)|^{2} d \theta .
$$

Therefore, in such a case, we can conclude that the least squares approach is equivalent to a linear system with circular symmetry, the MTF (that we call the average MTF, and the corresponding average image being given by

$$
\hat{K}(|\omega|)=\sqrt{\hat{H}(|\omega|)}, \quad \hat{g}_{a v}(\omega)=\frac{1}{2 \pi} \int_{0}^{2 \pi} \frac{\hat{K}_{\theta}^{*}(\omega)}{\hat{K}(|\omega|)} \hat{g}_{\theta}(\omega) d \theta .
$$

More generally, we can say that, if the band of the instrument does not change during the rotation (and this condition is satisfied also in Astronomy) then the band of the 
system, derived from the least-squares approach, is a circle, even if the MTF does not have a circular symmetry. But, hopefully, this lack of symmetry can be corrected by the deconvolution process.

In Fig. 4 we compare the cut of the average (symmetric) MTF with the cut of the original MTF along the direction of maximum resolution. In the left panel we show the case of LBT, assuming, as in Fig. 2, an ideal PSF (no aberration and no atmosphere). The profile of the average PSF is very similar to the profile of the original MTF along the baseline, showing that the maximum resolution is achieved in all directions. However, this result is obtained at the cost of a lowering of the MTF outside the central disc (corresponding to the band of a $8.4 \mathrm{~m}$ mirror), so that the price is an increase of the ill-conditioning of the deconvolution problem. A similar remark applies to the case of the confocal microscope, shown in the right panel. In conclusion, it may be difficult to achieve the optimal isotropic resolution by means of a least squares approach, which implies the use of an average image and a corresponding average MTF. A more accurate processing of the detected images is provided by the methods considered in the next section.

\section{MULTIPLE IMAGE DECONVOLUTION: RECONSTRUCTION METHODS}

In practice, we have only a finite set of images, corresponding to different orientations of the instrument with respect to the object. Since, as it is well known, image deconvolution is an ill-posed problem [7], an accurate modeling of the problem is required, including a statistical model of the noise, and, possibly, a priori information on the unknown object.

The images and the object consist of $N \times N$ arrays and are denoted by bold letters. We assume to have $p$ different images $\mathbf{g}_{1}, \mathbf{g}_{2}, \ldots, \mathbf{g}_{p}$, and we denote by $\mathbf{n}$ a multi-index (2D or $3 \mathrm{D})$ characterizing the pixels or voxels of the image. Then, if $\mathbf{g}_{j}(\mathbf{n}),(j=1, \ldots, p)$ is the value of one of these images at pixel $\mathbf{n}$, according to the model proposed by Snyder et al. ([19]) for images acquired with a CCD camera, we can write

$$
\mathbf{g}_{j}(\mathbf{n})=\mathbf{g}_{\mathrm{obj}, j}(\mathbf{n})+\mathbf{g}_{\text {back }, j}(\mathbf{n})+\mathbf{r}_{j}(\mathbf{n}),
$$

where $\mathbf{g}_{\mathrm{obj}, j}(\mathbf{n})$ is the number of photoelectrons due to object radiation; $\mathbf{g}_{\text {back }, j}(\mathbf{n})$ is the number of photoelectrons due to external and internal background, dark current, etc; $\mathbf{r}_{j}(\mathbf{n})$ is the read-out noise due to the amplifier. The first two terms are realizations of independent Poisson processes (photon noise), so that their sum is also a Poisson process, with expected value given by

$$
E\left\{\mathbf{g}_{\text {obj }, j}(\mathbf{n})+\mathbf{g}_{\text {back }, j}(\mathbf{n})\right\}=\left(\mathbf{K}_{j} * \mathbf{f}\right)(\mathbf{n})+\mathbf{b}_{j}(\mathbf{n}),
$$

where: $\mathbf{K}_{j}$ is the point spread function (PSF), corresponding to the $j$-th image; $\mathbf{f}$ is the object array, formed by the average numbers of photons emitted at the pixels/voxels of the object domain; $\mathbf{b}_{j}(\mathbf{n})$ is the expected value of the background. In the following we will denote by $A_{j}$ the matrix defined by the convolution product with the PSF $\mathbf{K}_{j}$ : $A_{j} \mathbf{f}=\mathbf{K}_{j} * \mathbf{f}$, and by $A_{j}^{T}$ the transposed matrix. Moreover we will assume that each PSF 
is normalized in such a way that the sum of their values is one. Finally the last term in Eq. (11) is the realization of an independent Gaussian process.

In the case of images dominated by photon noise, the read-out noise can be neglected and the likelihood function is given by the product of the likelihood functions of the different images, the latter being given by a product of Poisson distributions, one for each pixel/voxel of the image domain/volume. The maximization of this function is equivalent to the minimization of the Csiszár divergence (Csiszár [9]) given by

$$
\left.J_{0}(\mathbf{f} ; \mathbf{g})=\sum_{j=1}^{p} \sum_{\mathbf{n}}\left\{\mathbf{g}_{j}(\mathbf{n}) \ln \frac{\mathbf{g}_{j}(\mathbf{n})}{\left(A_{j} \mathbf{f}\right)(\mathbf{n})+\mathbf{b}_{j}(\mathbf{n})}+\left[\left(A_{j} \mathbf{f}\right)(\mathbf{n})\right)+\mathbf{b}_{j}(\mathbf{n})-\mathbf{g}_{j}(\mathbf{n})\right]\right\} .
$$

This functional is convex and non-negative, so it has global minima. Moreover it is not difficult to prove that there exists a unique minimum point if all the image values $\mathbf{g}_{j}(\mathbf{n})$ are strictly positive, a condition which is, in general, satisfied in the case of a sufficiently large background. Then the problem of object reconstruction can be formulated as the problem of minimizing this functional with the additional constraints of non-negativity and flux conservation

$$
\mathbf{f}(\mathbf{n}) \geq 0, \sum_{\mathbf{n}} \mathbf{f}(\mathbf{n})=c
$$

the constant $c$ being the average flux of photons coming from the object, i. e.

$$
c=\frac{1}{p} \sum_{j=1}^{p} \sum_{\mathbf{n}}\left\{\mathbf{g}_{j}(\mathbf{n})-\mathbf{b}_{j}(\mathbf{n})\right\} .
$$

An iterative method converging to a minimum point of the functional (the minimum point, in the case of uniqueness) can be obtained by means of the approach introduced by Shepp and Vardi [18], with a modification introduced by Lanteri et al. [12] for taking into account the flux constraint. The resulting algorithm is as follows

- choose an initial $\mathbf{f}^{(0)}>0$ such that its total flux coincides with $c$;

- given $\mathbf{f}^{(k)}$, compute

$$
\tilde{\mathbf{f}}^{(k+1)}=\mathbf{f}^{(k)} \sum_{j=1}^{p} A_{j}^{T} \frac{\mathbf{g}_{j}}{A_{j} \mathbf{f}^{(k)}+\mathbf{b}_{j}}, \tilde{c}^{(k+1)}=\sum_{\mathbf{n}} \tilde{\mathbf{f}}^{(k+1)}(\mathbf{n})
$$

- set

$$
\mathbf{f}^{(k+1)}=\frac{c}{\tilde{c}^{(k+1)}} \tilde{\mathbf{f}}^{(k+1)},
$$

where $c$ is the constant defined in Eq. (15), and $k$ denotes the iteration number. Moreover, as usual, products and quotients of arrays are defined pixel by pixel (or voxel by voxel).

This method is an extension to the multiple image case of the well-known RichardsonLucy (RL) method [16, 13], also known in tomography as the expectation-maximization (EM) method [18]. It is interesting to remark that the multiple image case has an interesting similarity with tomography, a tomographic projection being replaced by an image corresponding to a given direction. Thanks to this similarity an acceleration of the method, known in tomography as ordered subset expectation maximization (OSEM) 

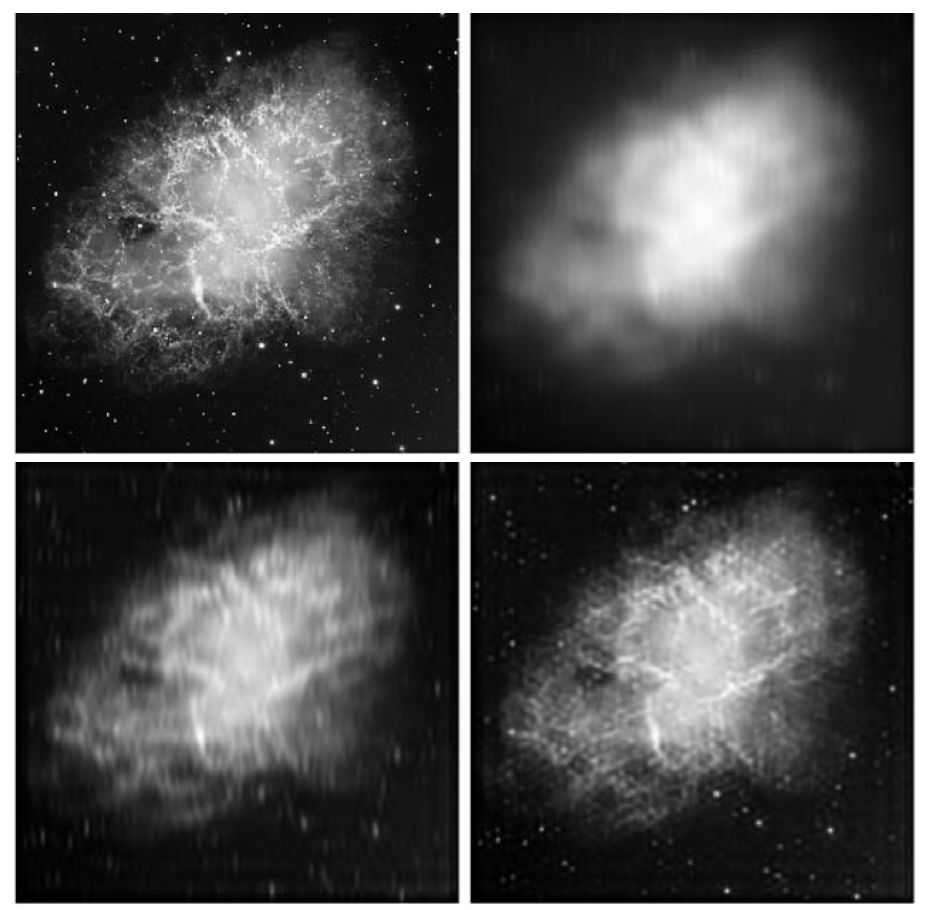

FIGURE 5. Example of reconstruction of a nebula (VLT/ESO image of M1). Left-upper panel: the object. Right-upper panel: the image corresponding to an orientation of the baseline of $0{ }^{\circ}$. Left-lower panel: the reconstruction of the nebula as derived from the previous image. Right-lower panel: the reconstruction obtained using three images with orientations of the baseline of $0^{\circ}, 60^{\circ}$, and $120^{\circ}$. About 100 OSEM iterations have been used.

[11], has been proposed for the reconstruction of LBT images [8]. The new algorithm can be obtained by replacing the previous algorithm by

- choose an initial $\mathbf{f}^{(0)}>0$ such that its total flux coincides with $c$;

- given $\mathbf{f}^{(k)}$, set $\mathbf{h}^{(0)}=\mathbf{f}^{(k)}$ and, for $j=1, \ldots, p$, compute

$$
\tilde{\mathbf{h}}^{(j)}=\mathbf{h}^{(j-1)} A_{j}^{T} \frac{\mathbf{g}_{j}}{A_{j} \mathbf{h}^{(j-1)}+\mathbf{b}_{j}}, \tilde{c}^{(j)}=\sum_{\mathbf{n}} \tilde{\mathbf{h}}^{(j)}(\mathbf{n}) ; \mathbf{h}^{(j)}=\frac{c}{\tilde{c}^{(j)}} \tilde{\mathbf{h}}^{(j)}
$$

- $\operatorname{set} \mathbf{f}^{(k+1)}=\mathbf{h}^{(p)}$.

In other words, one iteration of the multiple image RL method is replaced by one OSEM iteration, which consists of a cycle over the $p$ images, each step of the cycle being a single image RL iteration. About the computational cost, it is easy to see that one multiple image RL iteration requires the computation of $3 p+1$ FFTs, while one OSEM iteration requires the computation of $4 p$ FFTs. However, the number of OSEM iterations 
required for reaching the same result is smaller by a factor of $p$ than the number of RL iterations, so that the gain in computational cost is approximately a factor $(3 p+1) / 4$.

In Fig. 5, we give an example of reconstruction of an object obtained from the VLT/ESO image of the Crab Nebula M1. The LBT images have been obtained by convolving the object with three PSFs corresponding to orientations of $0^{\circ}, 60^{\circ}$, and $120^{\circ}$, assuming an AO correction of about $70 \%$ in K-band. As shown in the left-lower panel of the figure, if only the $0^{\circ}$ image is used for reconstructing the object (single image deconvolution), the result is clearly affected by the anisotropy in the resolution of the detected image. On the other hand the use of three images, combined with the OSEM algorithm, is already able to provide an image with an approximately isotropic resolution.

As it is known, the problem of minimizing the functional of Eq. (13) may be illposed: for instance, by pushing the iterations too far away one obtains the so-called checkerboard effect in the reconstruction of diffuse objects. A simple way to regularize the solution is to stop the iterations before too much degradation occurs. Alternatively, true regularization can be achieved by adding a penalty term to the Csiszár divergence

$$
J_{\mu}(\mathbf{f} ; \mathbf{g})=J_{0}(\mathbf{f} ; \mathbf{g})+\mu J_{R}(\mathbf{f}),
$$

where $\mu$ is the so-called regularization parameter. By applying a general approach, known as split gradient method (SGM) [12], it is possible to derive a simple modification of the RL method, converging to a minimum point of this functional. The basic point is the following decomposition of the gradient of the regularization functional

$$
-\nabla_{\mathbf{f}} J_{R}(\mathbf{f})=\mathbf{U}_{R}(\mathbf{f})-\mathbf{V}_{R}(\mathbf{f})
$$

where $\mathbf{U}_{R}, \mathbf{V}_{R}$ are positive arrays. Such a decomposition always exists even if it is not unique. Then, as shown in [1], the OS-version of the regularized algorithm is obtained by substituting the iteration step in Eq. (18) by

$$
\tilde{\mathbf{h}}^{(j)}=\frac{\mathbf{h}^{(j-1)}}{\mathbf{1}+\frac{\mu}{p} \mathbf{V}_{R}\left(\mathbf{h}^{(j-1)}\right)}\left(A_{j}^{T} \frac{\mathbf{g}_{j}}{A_{j} \mathbf{h}^{(j-1)}+\mathbf{b}_{j}}+\frac{\mu}{p} \mathbf{U}_{R}\left(\mathbf{h}^{(j-1)}\right)\right) .
$$

Further work on the applications of this method is in progress, for different kinds of regularization functionals.

\section{SUPER-RESOLUTION BY DATA INVERSION}

Super-resolution is a keyword widely used in several contexts and with quite different significations. In general, a super-resolving method/technique is a way for going beyond the diffraction limit of an optical instrument. It can be obtained by means of suitable hardware or software. In this section we only consider "super-resolution by data inversion", because we restrict the analysis to the case where super-resolution is achieved by means of suitable methods of image inversion/deconvolution.

Even in this restricted sense, there exists a wide literature on this topic. We only mention the "historical" papers of Toraldo di Francia [20] and Wolter [22], as well as a relatively recent review [6]. 
Resolution beyond the diffraction limit requires the extrapolation of the Fourier transform of the object outside the band of the optical instrument and, as remarked in [22], this is possible if the object has a finite extent because, in such a case, the Fourier transform is analytic. Since the extrapolation problem is ill-posed (in practice, very sensitive to noise propagation from the data to the solution), unlimited super-resolution is impossible in the presence of noise. A first attempt at estimating how much super-resolution can be achieved in practice was developed in [5], where it is shown that the amount of super-resolution is basically controlled by two parameters: the space-bandwidth product (SBP) and the signal-to-noise ratio (SNR). In the case of an optical instrument the SBP is approximately given by the ratio between the size of the object and the diffraction limit; then the results derived in [5] imply that super-resolution is feasible when this parameter is not much greater than one, namely in the case of compact and unresolved objects. On the other hand the amount of super-resolution increases with increasing the SNR. An estimate in terms of the photon flux is given by Lucy ([14],[15]).

According to the previous remarks, the development of a super-resolving method requires an estimate of the domain $\mathscr{D}$ of the object, to be used as a constraint in the reconstruction algorithm so that the total flux of the reconstructed object is concentrated within $\mathscr{D}$. Moreover, as it has been claimed by some authors, other constraints, such as non-negativity and flux conservation, may help in getting a super-resolving method. It is interesting to remark that the RL method (or the OSEM method in the multiple-image case) is well suited for the implemention of all these constraints. Indeed, non-negativity of the iterates is automatically guaranteed, as well as flux conservation (at least in the case of zero background). On the other hand, the constraint on the support of the object can be implemented because both in RL and OSEM the result of one iteration contains as a factor the result of the previous one; therefore, if the initial guess is zero at a given pixel, then the results of all the iterations will be zero at that pixel. We call such a property the localization property of these methods, in the sense that we can constrain the object to be localized in a given domain $\mathscr{D}$ by a suitable initialization of the iterations, taking a function which is constant over $\mathscr{D}$ and zero elsewhere.

We used this remark for developing a super-resolving method [3] that has been implemented in the software package AIRY (Astronomical Image Reconstruction in interferometrY), developed by our group (http://dirac.disi.unige.it), in collaboration with astronomers of Arcetri Observatory and Nice University, for the reconstruction of LBT images. It applies to the case where the astronomical target consists of unresolved objects (angular size of the order of the diffraction limit) separated by an angle considerably greater than the diffraction limit, so that there is no overlapping of their images. It follows that one can assume that there is only one of these unresolved objects, surrounded by empty sky, in the image to be processed. Since the domain $\mathscr{D}$ of the object is not known, one must attempt an estimate of $\mathscr{D}$ by means of a preliminary deconvolution of the images. Therefore our approach consists of the following steps.

Step 1 - Apply the RL or OSEM method to the detected images, using a constant array as initial guess and a number of iterations such that the reconstructed object is sufficiently well localized. Then, define the domain $\mathscr{D}$ of the object by identifying the pixels where the flux of the result is greater than a selected threshold (for instance some percent of its maximum value); alternatively one can take a disc with a diameter equal to the diffraction limit and containing most of the flux of the reconstructed object. 

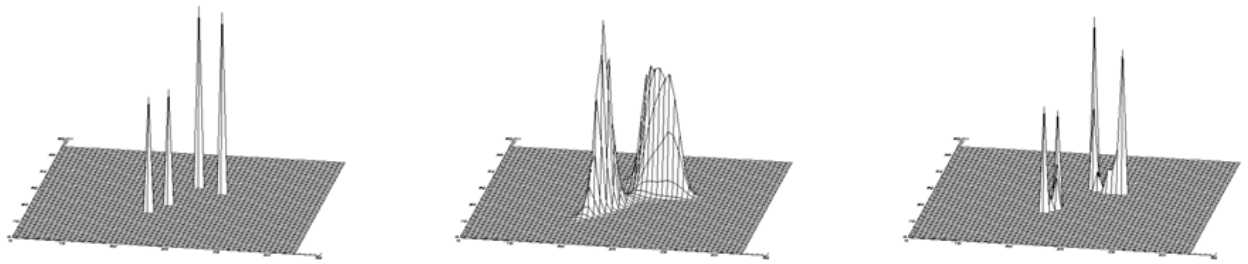

FIGURE 6. Left panel: a cluster of four stars with angular separation smaller than the diffraction limit. Middle panel: the reconstruction provided by OSEM initialized with a constant array (as before three images at $0^{\circ}, 60^{\circ}$, and $120^{\circ}$ are used). Right panel: the reconstruction provided by OSEM initialized with a suitable mask (see the text for details).

Step 2 - Apply again the RL or OSEM method to the detected images, but now initialized with the mask of the domain, namely a function which is constant over the domain and zero elsewhere. Each iterate is localized in the selected domain, and, after a suitable number of iterations, the point sources contained in the object can be separated.

In Fig. 6 we given an example of result obtained by means of this approach. The object, shown in the left panel, consists of a cluster of two binaries; within each binary the angular separation is $1 / 3$ of the diffraction limit while the separation between the centers of the two binaries is $2 / 3$. Moreover, within each binary the difference of magnitude is 0 while the difference of magnitude between the two binaries is 0.5 . Images have been obtained by convolving this object with ideal PSFs, and corrupting the result with background and noise. Observations at $0^{\circ}, 60^{\circ}$ and $120^{\circ}$ have been considered. As shown in the middle panel of the figure, after the first step the two binaries are resolved (an effect of the moderate super-resolution provided by OSEM) but the stars within the two binaries are not. This result is provided by the second step, as shown in the right panel. The mask used in this step has been obtained from the result of the first one by means of a threshold of about $50 \%$ of the maximum value.

\section{CONCLUDING REMARKS}

In this paper we investigated the case of optical instruments providing anisotropic resolution. Two examples, the Large Binocular Telescope and the confocal microscope, have been briefly described. Isotropic resolution can be achieved if it is possible to acquire different images of the same object corresponding to different orientations of the line of maximal resolution with respect to the object. Then multiple image deconvolution methods must be used for combining the different images into a unique one with isotropic resolution. In the two cases mentioned in this paper such a result can be achieved with a coverage of about $120^{\circ}$. The proposed deconvolution methods are related to the classical RL or EM methods. Moreover an approach leading to simple modifications of these methods, able to provide regularized solutions, is briefly discussed. 


\section{ACKNOWLEDGMENTS}

We give credit to the "Large Binocular Telescope Observatory" for Figure 1. We also thank our colleague and friend Christine De Mol for reading the paper and suggesting improvements.

\section{REFERENCES}

1. B. Anconelli, M. Bertero, P. Boccacci, M. Carbillet, and H. Lanteri, Proc. SPIE 5491, 932 (2004)

2. B. Anconelli, M. Bertero, P. Boccacci, M. Carbillet, and H. Lanteri, Astron. Astrophys 430, 731 (2005)

3. B. Anconelli, M. Bertero, P. Boccacci, M. Carbillet, Astron. Astrophys 431, 747 (2005)

4. J. R. P. Angel, J. M. Hill, P. A. Strittmatter, P. Salinari, and G. Weigelt, Pro. SPIE 3352, 881 (1998)

5. M. Bertero, and E. R. Pike, Optica Acta 29, 727 (1982)

6. M. Bertero, and C. De Mol, in Progress in Optics Vol. XXXVI, edited by E. Wolf, 129 (1996)

7. M. Bertero, and P. Boccacci, Introduction to Inverse Problems in Imaging, IoP Publishing, Bristol (1998)

8. M. Bertero, and P. Boccacci, Astron. Astrophys. S 144, 181 (2000)

9. I. Csiszár, Ann. Stat. 19, 2032 (1991)

10. R. Heintzmann, and C. Cremer, j. Microscopy, 7 (2002)

11. H. M. Hudson, and R. S. Larkin, IEEE Trans. Med. Im. 13, 601 (1994)

12. H. Lanteri, M. Roche, and C. Aime, Inverse Problems 18, 1397 (2002)

13. L. Lucy, Astron. J. 79, 745 (1974)

14. L. Lucy, Astron. Astrophys. 261, 706 (1992)

15. L. Lucy, Astron. J. 104, 1260 (1992)

16. W. H. Richardson, J. Opt. Soc, Am. 62, 55 (1972)

17. K. Sätzler, and R. Eils, Bioimaging 5, 171 (1997)

18. L. A. Shepp, and Y. Vardi, IEEE Trans. Med. Im. 1, 113 (1982)

19. D. L. Snyder, A. M. Hammoud, and R. L. White, J. Opt. Soc. Am. A10, 1014 (1993)

20. G. Toraldo di Francia, J. Opt. Soc. Am. 47, 497 (1955)

21. T. Wilson. Confocal Microscopy, Academic, New York (1990)

22. H. Wolter, Progress in Optics Vol. I, edited by E. Wolf, 155 (1961) 